\title{
The Dry-filling System for the Shock Absorber of Car Engine
}

\author{
Rong Du, Li Wen Zhang, Xiao Yan Liu ${ }^{1}$ \\ ${ }^{1}$ Hebei Jiaotong Vocational \& Technical College, China
}

\begin{abstract}
The shock absorber is considered as an important component of the suspension system. In its production process, the liquid filling for the shock absorber is a very important part. This paper introduced one kind of shock absorber stem -filling system, which achieved liquid dry -filling in the shock absorber for the car engine. The results showed that this system is stable and reliable.
\end{abstract}

\section{Introduction}

As an important component of the suspension system, the shock absorber of car engine ${ }^{[1]}$ plays a significant role on the passenger comfort, stability and safety. In its production process, the perfusion for the shock absorber is a very important part.

Perfusion is generally divided into wet-filling and dryfilling.Wet-filling means that the artificial materials will be filled into the liquid storage tank. Although providing the products with the vacuum environment, it causes damages to people due to the low production efficiency, the high production cost, and the direct contact with the liquid (ethylene glycol). At the same time the shell of the product will inevitably cause the liquid residues which influence the stability and security of the vehicles. The development and the manufacture of the shock absorber dry- filling equipment are introduced in the paper. The operation of the equipment is simple, improving the artificial operating environment. At the same time it can improve the production efficiency and the filling precision.

\section{The introduction of the system}

Dry-filling machine is used to extract the air from the cavity of shock absorber before the vacuum degree reach a certain requirement. Then it infuse the cavity with ethylene glycol, sealing it to ensure that there will be no bubbles in it. The equipment is shown in figure 1.

\section{The design of the mechanical parts ${ }^{[3]}$}

The mechanical parts of the system is mainly composed of a station indexing system, a vacuum system, infusion liquid, a residue recovery system and a pressure steel ball system.

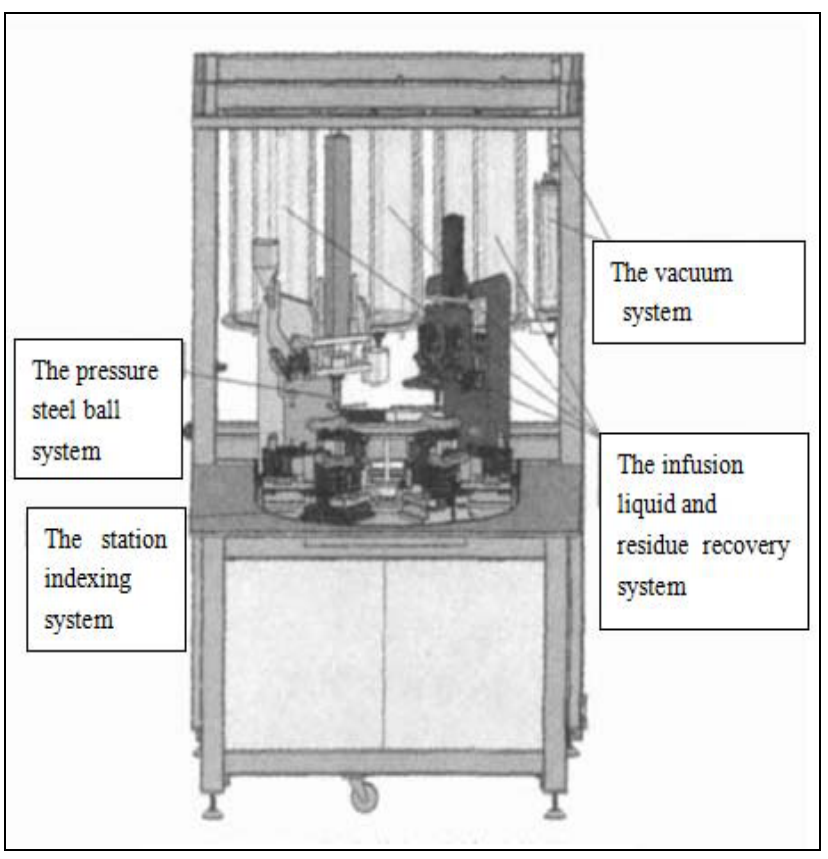

Figure 1. Dry-filling machine system structure.

\subsection{The station indexing system}

The dry-filling machine station indexing system is shown in figure 2.There are six stations in this part running by clockwise rotation. Each station reserves an interface for the series connection. When a product finishes its work in a certain station, the indexing plate rotating $1 / 6$ circle will make the products into the next station for processing. The control of the stepping motor makes the back wheel intermittent regular motion. The index plate rotation is driven by the cylindrical indexing cam mechanism. When the cam rotates, the dividing section contour will rotate the roller to make the indexing plate rotate. When the cam turn in, the rest period of the 
contour, the two adjacent rollers on the torus of the cam will make the rotary plate stop.

The dry-filling machine is in the automatic state. Each station has a memory function on whether there is a material (the feeding station have artifacts into), whether the artifacts are qualified (including the twice vacuumizing and the encapsulation of the pressure steel balls). So if the indexing plate rotates automatically one time, it will send the data to the workstation in turn.

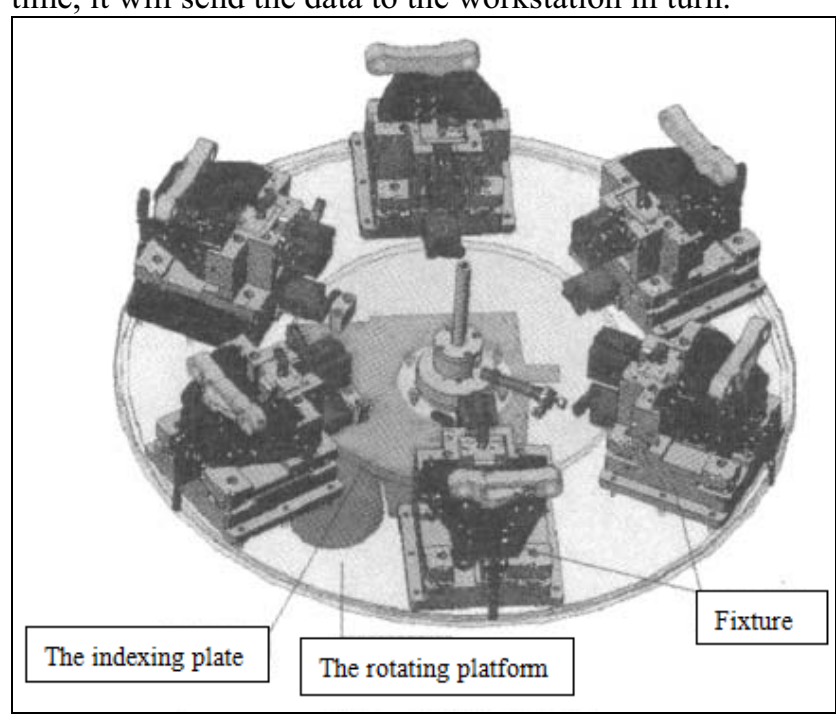

Figure 2. The station indexing system

\subsection{The vacuum, infusion liquid and the residue recovery system}

Above all, during the whole process of fluid infusion, we should ensure the vacuum degree ${ }^{[3]}$ to meet the requirements. The vacuum requirement refers to the best vacuum optimal value before the infusion vacuumizes the cavity of the product. Generally speaking, the higher the vacuum degree is, the less air bubbles inside the cavity there will be. At the same time, the function of the product will be better. But the high vacuum degree means the high cost of the filling machine. So our design manages to balance the three comprehensive optimal goals---the cost, the efficiency and the function.

The process of the vacuum uses the negative pressure.The exhaust tube controlled by the vacuum system begins to extract air, making the cavity of the product reach the certain vacuum degree.It forms a pressure difference between the cavity and the atmosphere. As a result, the liquid in the storage tank is filled into the cavity by its gravity caused by the atmospheric pressure inside. According to the requirement of the product specification, the first suction pipe of the system should achieve the vacuum degree for $800 \mathrm{~Pa}$ or less while the second suction pipe should reach the vacuum degree for $\mathrm{Pa} 200$ or less. After meeting the vacuum degree requirements, the ethylene glycol from both $\mathrm{A}$ and $\mathrm{B}$ liquid tanks will be infused into the cavity of the product.The excess liquid around the pipe mouth will be pumped into the residual liquid tank, and then returns to the $\mathrm{A} / \mathrm{B}$ tanks. The vacuum, the infusion liquid and the residue recovery system are shown in figure 3 .

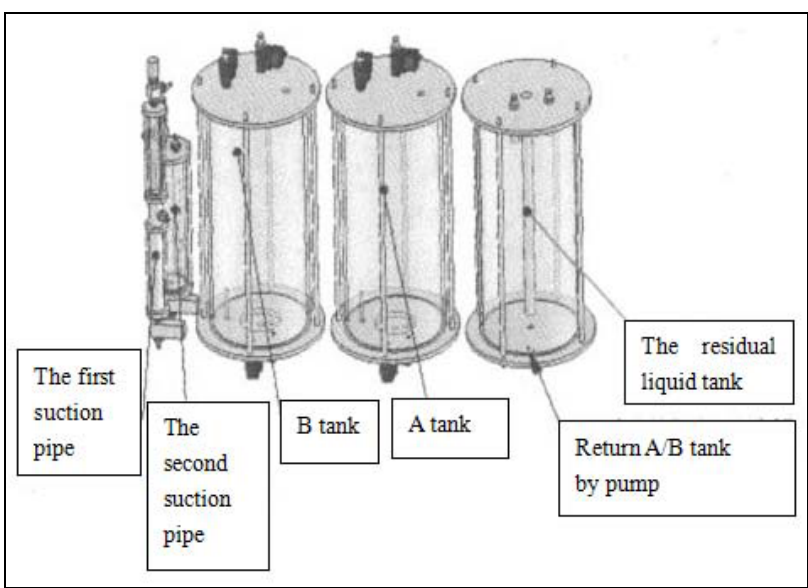

Figure 3. The vacuum, infusion liquid and the residue.

\subsection{The pressure steel ball system}

The structure diagram of the pressure steel ball system is shown in figure 4. After filling with the ethylene glycol and waiting for the workpiece in place, the TOX cylinder will seal it with the steel ball. The residual ethylene glycol around the steel ball will be recycled to the residual liquid tank at the instant of the movement of the pressure steel ball. The seal must be safe in this section to ensure that there will be no leakage and no escape of the steel balls. The riveting depth of the steel ball should meet the requirements. The depth can be also displayed by the displacement sensor. In this station, the steel ball is automatic feeding through the steel ball pipe to the filter base, ensuring that just one steel ball can go through every time. With the use of the optical fiber sensor, we can detect the existence of the steel balls. The PLC will alarm when there are no balls.

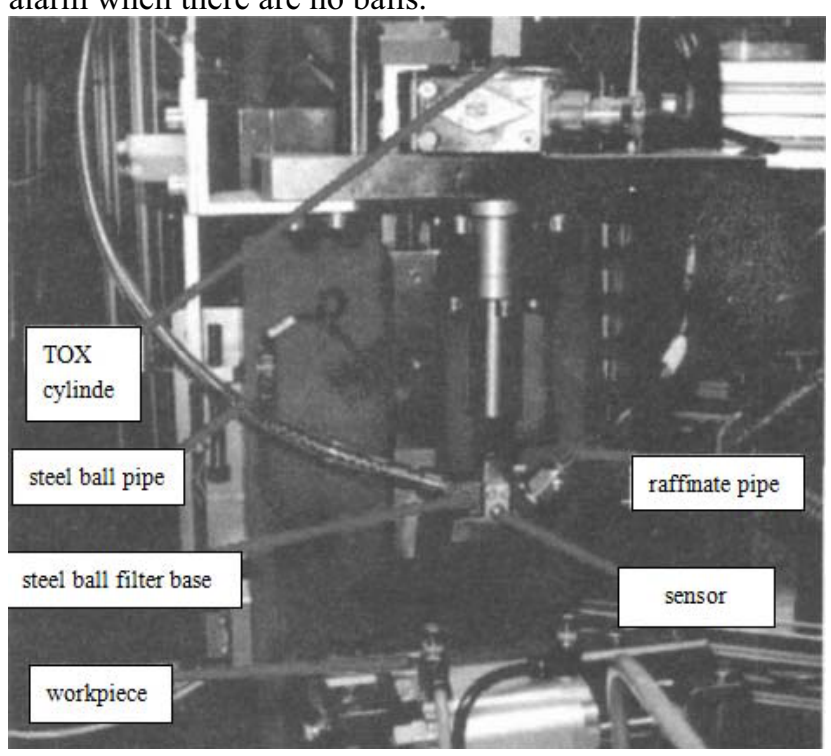

Figure 4. Pressure steel ball system.

\section{The design of the control section}

Due to the complicated control process and the various data,this equipment uses the IPC + PLC (industrial personal computer + programmable logic controller) to 
control the model of the upper and lower place machine. The PLC can control the station motions. And the industrial computer charges the whole course of the monitoring information to achieve the actions in the process of production control, field monitoring and data processing.

\subsection{The hardware design of the control part}

As the core part of the control part hardware,the Mitsubishi FX2N-80MT is a programmable controller, which has low cost, high efficiency and powerful functions. The industrial personal computer is used by an ADVANTECH PCI-1711L bus data acquisition card.It is a powerful low cost, multifunction PCI bus data acquisition card,using programmable single-ended channel input mode.The sensor can change the nonelectric signals such as the displacement,the pressure,the temperature and the vacuum degree into the electric signal and PCI-1711L converts the input analog voltage into a digital signal and then gives it to the industrial personal computer to process. (figure 5).

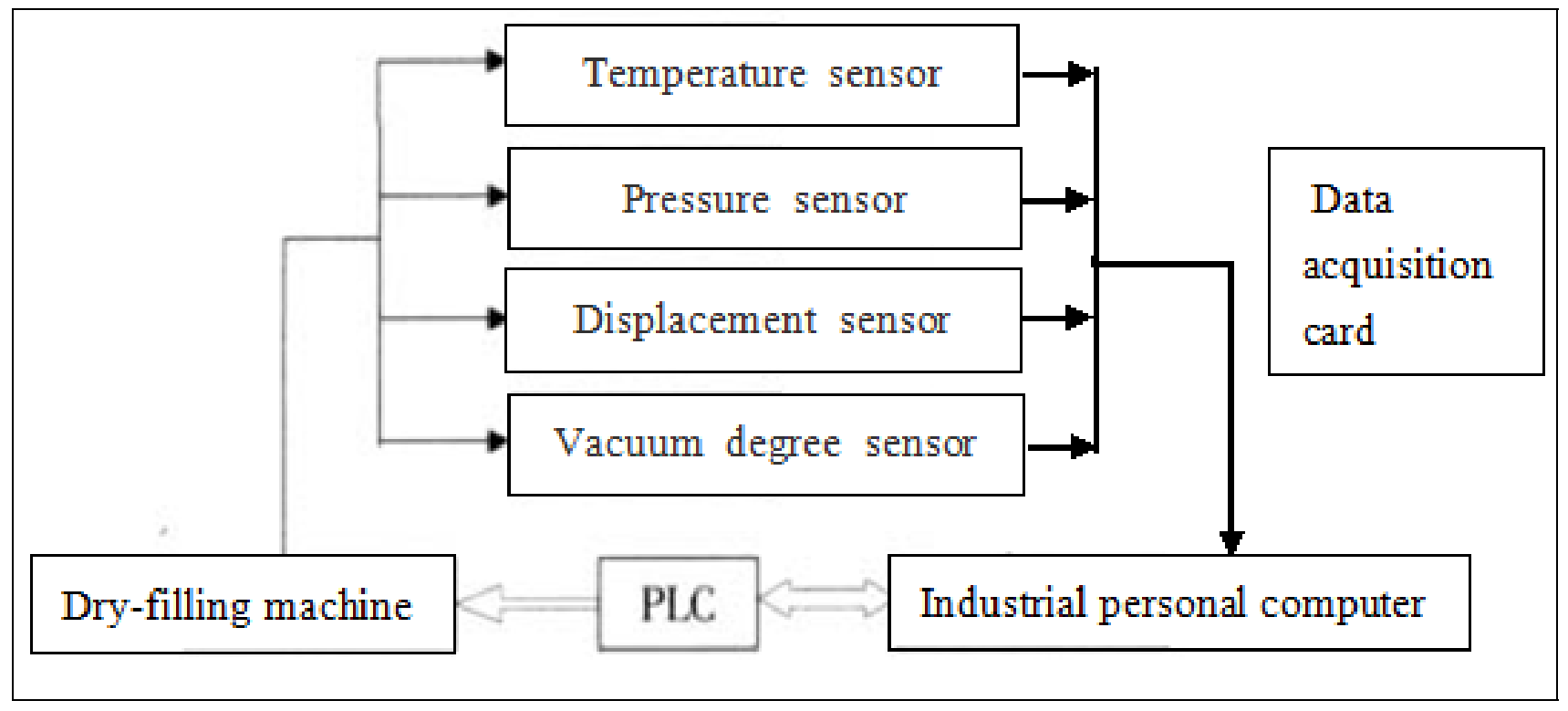

Figure 5. The hardware structure of the control part

\subsection{The software design of control part}

\subsubsection{The system process}

The flow chart of the whole system is shown in figure 6. When the equipment starts, the system will be initialized.Various parameters set up by the automatic mode feeding station begin to feed.With material in the process of the vacuum system and the infusion liquid twice vacuumize the cavity of the product,it ensures that there is no bubbles after the infusion liquid fills into cavity. When the vacuum degree meets the requirements, the infuse quantitative ethylene glycol will fill into the cavity.After filling the infusion liquid,the residue on the surface of the product will be extracted back in residual liquid tank and seal the product pouring mouth with equal diameter steel ball. When the product meets the requirement,the setting parameters will be qualified,otherwise the system will alarm and the detailed information will be shown in the master control page.

\subsubsection{The design of the interface}

The equipment control interface uses LabVIEW $^{[4]}$ software design .LabVIEW is a general programming system, with powerful database, including the serial instrument control, the data acquisition, the data analysis, the data display and the data storage. System monitoring interface of the device is mainly composed of the vacuum and infusion station monitoring, the pressure steel ball station monitoring pressure, the discharge station monitoring, the liquid level monitoring, the fault information system, the system status display, and the system operation control (figure 7). The interface can see visually dynamic vacuum degree online after the shock absorber in the vacuum process. It can also see the curve of reflected changes about the shock absorber vacuum degree .

\subsubsection{Data processing}

The LabVIEW software provides a variety of ways to data storage and reading. In addition to save the data, the test data file can generate the data produced by the information such as the date, the time and the parameters of each product, making the users check the data at a certain moment easily. Figure 8 is a data query interface, we can get the information of the product by entering a query condition. 


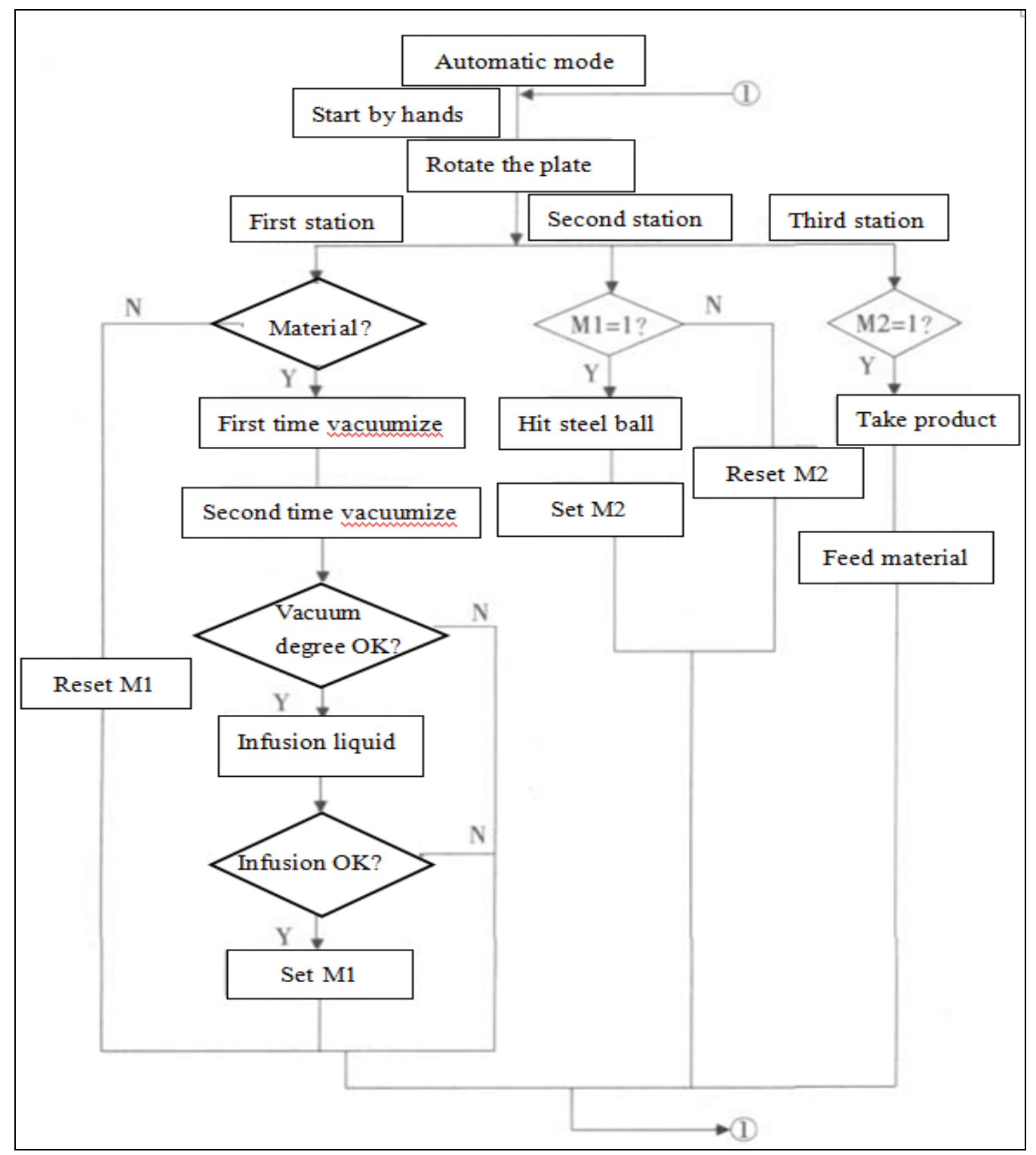

Figure 6. The flow chart of the system.

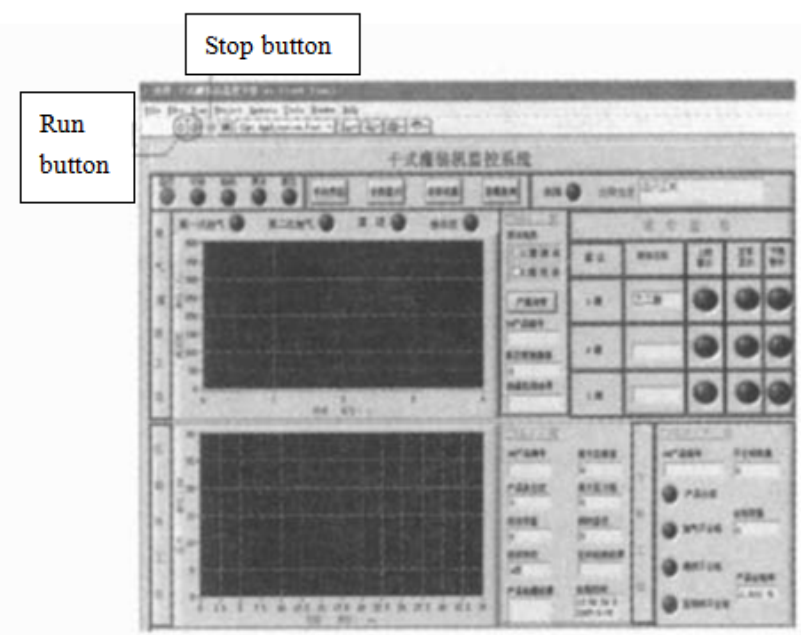

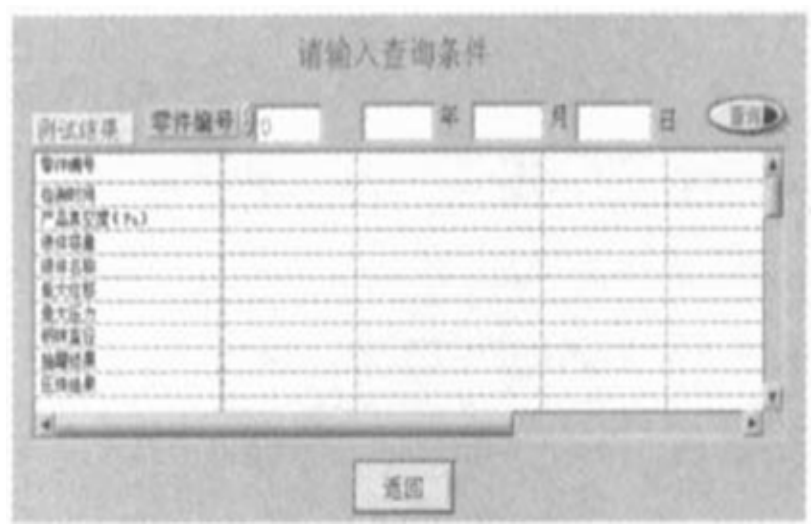

Figure 8. Data inquiry interface.

Figure 7. System monitor interfaces. 


\section{Conclusion}

The shock absorber ${ }^{[5]}$,as an important vehicle part, has high technical content.Its technical level reflects the development level of China's auto parts to some extent.The engine suspension shock absorber dry filling equipment introduced in this paper has been put into use in a suspension system co.,LTD. The design and the process of the equipment have fully considered the influence of various factors on the quality of the shock absorber filling. Thus, not only does it improve the work efficiency in the actual production, but also improve the operating environment of the workers.At the same time,the filling precision of the products has also reached a higher level.

\section{References}

1. Deqing Zhang,Modeling and Simulation Design of Vehicle Shock Absorber. Zhenjiang: Jiangsu University(2003).

2. Hao Xu,Chunyuan Cai,Junqi Yan,etc.Mechanical Design Handbook.Beijing: Mechanical Industry Press(2001).

3. Zuotong Liu.Vacuum Technology and Equipment.Nanjing:Southeast University Press(1991).

4. 4.Zhenshan Lei.LabVIEW7 Express Practical Technology Course. Beijing: China Railway Press(2005).

5. Xuemei Feng, Zuomin Liu. Development and Current Situation of Automotive Hydraulic Shock Absorber Technology .Journal of Wuhan University of Technology,2003(6):340-342 